\title{
Erratum to: Intermediary Liability for IP Infringement in Finland: CopyRight vs. CopyLeft-A Series of Legislative Proposals and Decade of Debates
}

\author{
Katja Lindroos
}

Erratum to:

Chapter 8 in: G.B. Dinwoodie (ed.), Secondary Liability of Internet Service Providers, Ius Comparatum - Global Studies in Comparative Law 25, https://doi.org/10.1007/978-3-319-55030-5_8

The original version of this book was published with incorrect Chapter title in Chapter 8.

It has been corrected and updated as:

Intermediary Liability for IP Infringement in Finland: CopyRight vs. CopyLeft-A Series of Legislative Proposals and Decade of Debates

The online version of the updated original chapter can be found at https://doi.org/10.1007/978-3-319-55030-5_8 